\title{
Diabetic groups as defined by ADA and NDDG criteria have a similar aerobic capacity, blood pressure and body composition
}

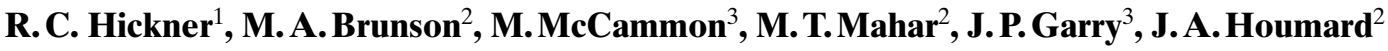 \\ ${ }^{1}$ Human Performance Laboratory, Department of Exercise and Sport Science, and the Department of Physiology, East Carolina \\ University, Greenville, North Carolina, USA \\ ${ }^{2}$ Human Performance Laboratory, Department of Exercise and Sport Science, East Carolina University, Greenville, \\ North Carolina, USA \\ ${ }^{3}$ Department of Family Medicine, East Carolina University, Greenville, North Carolina, USA
}

\section{Abstract}

Aims/hypothesis. In 1997 the American Diabetes Association (ADA) published new categories for diabetes based on fasting plasma glucose that classified diabetes as a plasma glucose of $7.0 \mathrm{mmol} / \mathrm{l}$, or more, rather than one of $7.8 \mathrm{mmol} / \mathrm{l}$ or more, as published previously by the National Diabetes Data Group (NDDG) in 1979. We compared the cardiovascular disease risk factors of subjects classified as having Type II (non-insulin-dependent) diabetes mellitus under the NDDG and ADA criteria.

Methods. We examined a database of approximately 3700 men ( $40.4 \pm 11.5$ years old) and distributed them into four categories: normal fasting plasma glucose (NFG) of less than $6.1 \mathrm{mmol} / 1$, impaired (IFG) 6.1 to $7.0 \mathrm{mmol} / \mathrm{l}, \mathrm{ADA}$ diabetic 7.0 to $7.8 \mathrm{mmol} / \mathrm{l}$ and NDDG diabetic of $7.8 \mathrm{mmol} / \mathrm{l}$ or more.

Results. Fasting glucose was $5.2 \pm 0.5,6.4 \pm 0.2$, $7.3 \pm 0.2$ and $11.2 \pm 2.9 \mathrm{mmol} / \mathrm{l}$ for the subjects of the NFG, IFG, ADA and NDDG groups, respectively. Estimated treadmill $\mathrm{VO}_{2 \max }$ was $41.4 \pm 8.0,36.0 \pm$ $7.8,32.2 \pm 7.6,30.6 \pm 7.0 \mathrm{ml} \cdot \mathrm{kg}^{-1} \cdot \mathrm{min}^{-1}$ in the NFG,
IFG, ADA, and NDDG groups, respectively (NFG and IFG > ADA and NDDG: $p<0.05$ ). The ADA and NDDG groups were also similar for resting and exercise blood pressure and body composition. Triglycerides and total: HDL cholesterol ratios were higher and LDL cholesterol concentration was lower, in the NDDG group than in all other groups $(p<0.05)$. Total and LDL cholesterol in the ADA and NDDG groups were similar.

Conclusion/interpretation. The similarities in the aerobic capacities, blood pressure and body composition of the ADA and NDDG groups indicate that the decision to lower the cut-off from $7.8 \mathrm{mmol} / \mathrm{l}$ to $7.0 \mathrm{mmol} / \mathrm{l}$ blood glucose for the clinical classification of diabetes was appropriate. The ADA and NDDG groups, however, might not have identical risks for cardiovascular disease because of differences between total:HDL cholesterol ratios, circulating HDL cholesterol and triglyceride concentrations. [Diabetologia (2001) 44: 26-32]

Keywords Cholesterol, exercise, glucose, hypertension, insulin.
Received: 4 May 2000 and in revised form: 7 September 2000

Corresponding author: R.C. Hickner, Human Performance Laboratory, 371 Ward Sports Medicine Building, East Carolina University, Greenville, NC 27858, USA

Abbreviations: ADA, American Diabetes Association; NDDG, National Diabetes Data Group; NFG, normal fasting glucose; $\mathrm{VO}_{2 \max }$, maximum aerobic capacity; WHO, World Health Organization; CDRFIRP, cardiovascular disease risk factor identification/reduction programme; FVC, forced vital capacity; $\mathrm{FEV}_{1}$, forced expiratory volume; ANCOVA, analysis of covariance.
In 1979 the National Diabetes Data Group (NDDG) published the classification system for diagnosing Type II (non-insulin-dependent) diabetes mellitus. This classification system consisted of two groups based on fasting plasma glucose: normal plasma glucose $(<6.4 \mathrm{mmol} / \mathrm{l})$ and diabetic $(\geq 7.8 \mathrm{mmol} / \mathrm{l})$. A person would, however, also be classified as diabetic if their $2 \mathrm{~h}$ plasma glucose during an oral glucose tolerance test was greater than $11.1 \mathrm{mmol} / \mathrm{l}$. In 1997 the American Diabetes Association (ADA) developed new categories for diabetes based only on fasting plasma glucose and classified diabetes as a fasting 
plasma glucose of $7.0 \mathrm{mmol} / \mathrm{l}$ or more [1]. This new classification system was implemented for the early detection and treatment of this disease. It is of considerable importance that subjects with fasting plasma glucose of 7.0 to $7.8 \mathrm{mmol} / \mathrm{l}$ have a cardiovascular disease risk profile similar to the group of subjects previously classified as diabetic (those with a fasting plasma glucose $\geq 7.8 \mathrm{mmol} / \mathrm{l}$ ) under the NDDG category. The new ADA criteria have, therefore, come into question both in the United States and in Europe [2-6].

Our purpose was to compare risk factors for cardiovascular disease (blood pressure, whole-body and regional adiposity and blood lipid profile) in subjects classified as Type II diabetic under the NDDG classification criteria (fasting plasma glucose $\geq 7.8 \mathrm{mmol} / \mathrm{l}$ ) with those who fall below the $7.8 \mathrm{mmol} / \mathrm{l}$ fasting glucose criteria but who are now classified as diabetic (subjects with fasting plasma glucose $<7.8$ but $\geq 7.0 \mathrm{mmol} / \mathrm{l}$ ) under the new ADA system. Although reduced aerobic capacity was found to correlate with increased risk of cardiovascular disease the relation between the new diabetes diagnostic criteria and reduced aerobic capacity has not been investigated [7]. We aimed to evaluate the relation between aerobic capacity and categorization of Type II diabetes.

\section{Subjects and methods}

We examined a database from the Cardiovascular Disease Risk Factor Identification and Reduction Programme (CDRFIRP) in the Human Performance Laboratory of East Carolina University. The database consisted of approximately 3700 men (40.4 \pm 11.5 years: $90 \%$ Caucasian, 9\% African American, and $<1 \%$ other). We distributed these subjects into four separate groups: normal fasting plasma glucose (NFG) less than $6.1 \mathrm{mmol} / \mathrm{l}(n=3307)$, impaired fasting glucose (IFG) $6.1-7.0 \mathrm{mmol} / \mathrm{l}(n=327)$, ADA diabetic 7.0 to $7.8 \mathrm{mmol} / \mathrm{l}(n=39)$ and NDDG diabetic greater than or equal to $7.8 \mathrm{mmol} / \mathrm{l}(n=72)$ based only on a resting, seated plasma glucose that had been obtained from each subject after a 12-h overnight fast. The prevalence of undiagnosed diabetes was therefore $1.0 \%$ in the ADA group and $1.9 \%$ in the NDDG group in this database. These prevalences of diabetes are slightly lower than those $(\sim 3 \%)$ of undiagnosed diabetic subjects in the United States $[8,9]$. The ADA diabetic group used in this study consists only of subjects with fasting plasma glucose from 7.0 to $7.8 \mathrm{mmol} / \mathrm{l}$. Thus there is no overlap between our ADA and NDDG groups. Subjects participated in the programme approved by the University after giving their informed consent. These subjects were not recruited specifically for this study but took part in a self-referral screening programme. They were mainly from eastern North Carolina, and without known or suspected cardiovascular, pulmonary or metabolic disease. They are, therefore, more likely to be healthy than the general population of eastern North Carolina. People enroll voluntarily for testing at the CDRFIRP either independently or as part of their employers' corporate health programme.
Body composition and anthropometric determinations. The per cent body fat was measured using hydrodensitometry as described previously [10]. Circumferences of the waist (umbilicus) and the hip (greater trochanter) were measured for to calculate waist-to-hip ratios. The circumferences were measured to the nearest $0.5 \mathrm{~cm}$ with a spring-loaded tape when subjects ended a normal expiration and were in a standing position. If duplicate measurements were not within at least $1.0 \mathrm{~cm}$, a third measurement was taken. The two closest measures were averaged and that average value was used in the data analysis.

Pulmonary function testing. To measure forced vital capacity, subjects were seated comfortably in a chair with a noseclip in place and were instructed to exhale slowly but to the maximum. When their residual volume had been reached they were encouraged to breathe in as deeply as possible. After a full inhalation, subjects breathed out all air as fast as possible (to maximum expiration) through a mouthpiece into a spirometer (Spiromate AS-600, Rikko Scientific and Medical Instruments, Fort Lee, N.J., USA). After a full expiration, they were instructed to inhale maximally again. Forced vital capacity (FVC) was the volume of air moved by the subjects from maximum inhalation to maximum expiration. The per cent forced expiratory volume $\left(\mathrm{FEV}_{1} / \mathrm{FVC}\right)$ was calculated by dividing the volume of air expired in the first $1 \mathrm{~s}\left(\mathrm{FEV}_{1}\right)$ by total forced vital capacity.

Maximal aerobic capacity. After resting 12-lead electrocardiogram (ECG) and blood pressure monitoring, subjects underwent a graded maximum stress test on a motorized treadmill using Balke or Bruce protocols. The protocol used depended on the physical ability of the subject. Heart rate and ECG (12-lead, Quinton Q5000, Quinton Instruments, Seattle, Wash., USA) and blood pressure were monitored throughout the test. The subjects exercised to volitional exhaustion. Subjects never listed orthopedic limitation (acute or chronic injury to the lower limbs) as the cause for terminating the test. The $\mathrm{VO}_{2 \max }$ was calculated from the speed and grade of the treadmill over the final stage of the exercise using reported prediction equations [11].

Statistical analysis. One-way analysis of variance (ANOVA) was done for each variable to examine potential differences in the NFG, IFG, ADA and NDDG groups. Tukey's post hoc test was used if significance was attained during the ANOVA. We ran an analysis of covariance (ANCOVA) to compare groups and because smoking is known to be a risk factor for peripheral arteriosclerosis and other cardiovascular disease, the variable of smoking (categorized as smoker vs non-smoker) was controlled $[12,13]$. The assumption that regression slopes are homogenous was tested on all ANCOVAs. No variables violated the assumption with the exception of aerobic capacity; therefore, ANCOVA was not carried out on this variable. The ANOVA was, however, done for aerobic capacity on all subjects, including non-smokers only (excluding the 787 smokers). These analyses on aerobic capacity excluded those with pulmonary limitations $(\mathrm{FVC}<2.0$ litres and $\mathrm{FVCl}$ $\mathrm{FEV}_{1}<60 \% ; n=142$ or $\sim 4 \%$ for each glucose group). Pulmonary limitations and smoking status were therefore controlled for the analysis of aerobic capacity. Pearson product moment correlations were carried out, controlling for age, between maximum heart rate, pulmonary function and maximum aerobic capacity. Results are given as means $\pm \mathrm{SD}$ and a $p$ value of less than 0.05 was considered significant. 
Table 1. Body composition and anthropometry

\begin{tabular}{lcccc}
\hline & NFG & IFG & ADA & NDDG \\
& $n=3307$ & $n=327$ & $n=39$ & $n=72$ \\
\hline Height (cm) & 177.8 & 178.3 & 178.2 & 177.9 \\
& 6.6 & 6.7 & 7.4 & 8.0 \\
Body weight (kg) & $85.7^{\mathrm{a}}$ & $91.0^{\mathrm{b}}$ & 96.0 & 99.9 \\
& 11.4 & 17.3 & 19.0 & 21.4 \\
BMI & $27.1^{\mathrm{a}}$ & $28.6^{\mathrm{b}}$ & 30.1 & 31.4 \\
& 4.0 & 5.0 & 5.2 & 5.7 \\
Body fat (\%) & $22.8^{\mathrm{a}}$ & $26.5^{\mathrm{b}}$ & 28.1 & 29.3 \\
hydrostatic & 6.8 & 7.2 & 6.6 & 7.2 \\
Waist (cm) & $94.4^{\mathrm{a}}$ & $99.8^{\mathrm{b}}$ & 104.0 & 105.8 \\
circumference & 10.7 & 13.2 & 12.6 & 14.4 \\
Hip (cm) & $95.7^{\mathrm{a}}$ & 101.3 & 102.3 & 105.0 \\
circumference & 9.9 & 11.0 & 15.9 & 13.6 \\
Waist-to-hip ratio & 0.99 & 0.99 & 1.02 & 1.01 \\
& 0.06 & 0.05 & 0.05 & 0.06 \\
\hline
\end{tabular}

Data are means \pm SD.

${ }^{a}$ Different from all other groups

${ }^{\mathrm{b}}$ Different from NDDG

\section{Results}

Body composition and anthropometry. The per cent of body fat, BMI and body weight were higher in the IFG, NDDG and ADA groups than in the NFG group $(p<0.05$; Table 1$)$. Waist and hip circumferences were smaller in the NFG groups than all others $(p<0.05)$ but there were no statistically significant differences between the groups in waist-to-hip ratio. There were also no statistically significant differences between IFG, NDDG and ADA groups in any of the body composition or anthropometric variables (Table 1).

Fasting plasma glucose. Fasting plasma glucose was $5.2 \pm 0.5,6.4 \pm 0.2,7.3 \pm 0.2$, and $11.2 \pm 2.9 \mathrm{mmol} / 1$ in NFG, IFG, ADA, and NDDG, respectively $(p<0.01)$. All groups were significantly different from one another because fasting plasma glucose was the basis for categorizing the groups $(p<0.01)$.

Treadmill stress test (maximum aerobic capacity, maximum heart rate and ECG). Maximum aerobic capacity $\left[\left(\mathrm{VO}_{2 \max }\right)\right.$ data expressed by kilogram body mass $\left.\left(\mathrm{ml} \cdot \mathrm{kg}^{-1} \cdot \mathrm{min}^{-1}\right)\right]$ are shown in Figure $1 \mathrm{~A}$. The $\mathrm{VO}_{2 \max }$ in NFG and IFG were higher $(p<0.05)$ than $\mathrm{VO}_{2 \max }$ values attained by ADA and NDDG. There was no statistically significant difference between ADA and NDDG $\mathrm{VO}_{2 \max }$. Absolute $\mathrm{VO}_{2 \max }$ data $\left(1 /\right.$ min) are shown in Figure $1 \mathrm{~B}$. The $\mathrm{VO}_{2 \max }$ in $\mathrm{NFG}$ was higher $(p<0.05)$ than the $\mathrm{VO}_{2 \max }(1 / \mathrm{min})$ values in all other groups. There was no statistically significant difference in $\mathrm{VO}_{2 \max }(\mathrm{l} / \mathrm{min})$ between the IFG, ADA and NDDG groups.

Maximum heart rates were $182.4 \pm 0.3$, $172.6 \pm 1.11,160.0 \pm 28.0$, and $163.6 \pm 18.6$ beats a

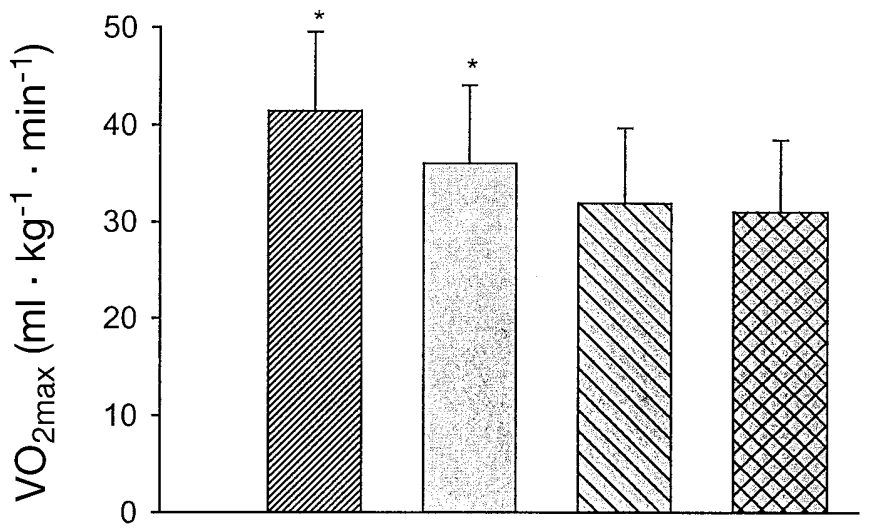

A

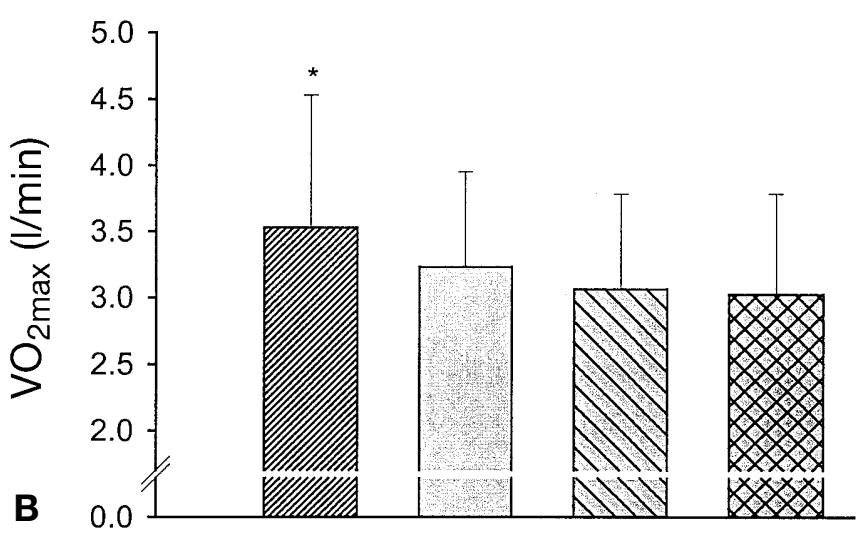

Fig.1A, B. Estimated maximum aerobic capacity $\left(\mathrm{VO}_{2 \max }\right)$ expressed by kilogram body weight $\left(\mathrm{ml} \cdot \mathrm{kg}^{-1} \cdot \mathrm{min}^{-1} \mathbf{A}\right)$ or in absolute terms (1/min: B) for subjects with normal fasting glucose (NFG, - impaired fasting glucose (IFG, $\square$ ), fasting plasma glucose $>7.0$ but $<140 \mathrm{mmol} / \mathrm{l}$ (ADA, $\mathbb{N}$ ), fasting plas-

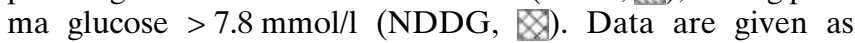
means \pm SD. * Significantly different from all other groups $(p<0.05)$

min in the NFG, IFG, ADA and NDDG groups, respectively $(p<0.05)$. Maximum heart rate in the ADA and NDDG groups was not different from one another, although the maximum heart rate in the ADA and NDDG groups was lower than in the IFG and NFG groups $(p<0.01)$. Partial correlation coefficients for the relation between maximum heart rate and $\mathrm{VO}_{2 \max }$, after controlling for age, were 0.317 , $0.466,0.341$ and 0.531 in the NFG, IFG, ADA and NDDG groups, respectively ( $p<0.05$ for all correlations).

Abnormal ECG responses, as indicated by ST segment depression greater than or equal to $1 \mathrm{~mm}$ past Jpoint or exercise-induced arrhythmias, were noted during the treadmill test in 105 subjects. There were 81 (or $2.5 \%$ of the NFG group) of these subjects in the NFG group, 11 (or $3.5 \%$ of the IFG group) in the IFG group, 4 (or $10.5 \%$ of the ADA group) in the ADA group and 9 (or $13.4 \%$ of the NDDG group) in the NDDG group. 
Table 2. Blood cholesterol and triglyceride content

\begin{tabular}{lllll}
\hline & NFG & IFG & ADA & NDDG \\
\hline Total & $5.27^{\mathrm{a}}$ & 5.64 & 5.64 & 5.74 \\
& 1.01 & 1.07 & 1.07 & 1.53 \\
HDL & 1.08 & 1.06 & 1.08 & 0.96 \\
& 0.28 & 0.30 & 0.26 & 0.32 \\
LDL & $3.49^{\mathrm{a}}$ & 3.77 & 3.58 & 3.63 \\
& $0.87^{\mathrm{a}}$ & 1.00 & 0.70 & 0.80 \\
Total: HDL & $5.17^{\mathrm{b}}$ & 5.75 & 5.5 & $6.58^{\mathrm{a}}$ \\
& $1.77^{\mathrm{a}}$ & 1.84 & 1.68 & 2.92 \\
Triglyceride & $1.56^{\mathrm{b}}$ & 1.86 & 1.77 & $2.85^{\mathrm{a}}$ \\
& $1.67^{\mathrm{N}}$ & 1.28 & 0.87 & 2.56 \\
\hline
\end{tabular}

Data are presented in units of $\mathrm{mmol} / \mathrm{l}$ and are means $\pm \mathrm{SD}$

${ }^{a}$ Different from all other groups

${ }^{\mathrm{b}}$ Different from IFG and NDDG

Table 3. Blood pressure

\begin{tabular}{lcccc}
\hline & NFG & \multicolumn{1}{c}{ IFG } & ADA & NDDG \\
\hline Resting systolic & $124.6^{\mathrm{a}}$ & $130.2^{\mathrm{b}}$ & 135.8 & 135.6 \\
& 12.0 & 15.1 & 8.5 & 14.6 \\
Resting diastolic & $78.6^{\mathrm{a}}$ & 81.4 & 84.5 & 84.7 \\
& $9.0^{\mathrm{a}}$ & 10.4 & 12.2 & 9.4 \\
Peak systolic & $190^{\mathrm{a}}$ & 196.5 & 199.8 & 199.6 \\
& 21.2 & 20.8 & 27.5 & 20.6 \\
Peak diastolic & $79.4^{\mathrm{a}}$ & 84.3 & 87.1 & 86.2 \\
& 10.7 & 10.9 & 13.8 & 12.8 \\
\hline
\end{tabular}

Data are presented as $\mathrm{mmHg}$ and are means \pm SD.

${ }^{a}$ Different from all other groups

${ }^{\mathrm{b}}$ Different from ADA and NDDG

Pulmonary function. Resting forced vital capacity (FVC) was $4.88 \pm 0.89,4.76 \pm 0.88,4.22 \pm 0.89$ and $4.19 \pm 0.94$ litres in the NFG, IFG, ADA and NDDG groups, respectively $(p<0.01)$. The FVC in ADA and NDDG groups were the same, although FVC in the ADA and NDDG groups were lower than in the IFG and NFG groups $(p<0.01)$. There was a positive relation between $\mathrm{FVC}$ and $\mathrm{VO}_{2 \max }$. Partial correlation coefficients for the relation between FVC and $\mathrm{VO}_{2 \max }$ in the study sample were 0.368 and 0.277 for relative $\left(\mathrm{ml} \cdot \mathrm{kg}^{-1} \cdot \mathrm{min}^{-1}\right)$ and $\mathrm{ab}-$ solute $(1 / \mathrm{min}) \mathrm{VO}_{2 \max }$, respectively $(p<0.01)$. The forced expiratory volume $\left(\mathrm{FEV}_{1}\right)$ was $3.92 \pm 0.70$, $3.72 \pm 0.71,3.27 \pm 0.67$ and $3.35 \pm 0.76$ litres in the NFG, IFG, ADA and NDDG groups, respectively $(p<0.01)$. The $\mathrm{FEV}_{1}$ in ADA and NDDG groups was the same and lower than in the IFG and NFG groups $(p<0.01)$. There was a positive relation between $\mathrm{FEV}_{1}$ and $\mathrm{VO}_{2 \text { max }}$. Partial correlation coefficients for the relation between $\mathrm{FEV}_{1}$ and $\mathrm{VO}_{2 \max }$ were 0.390 and 0.304 for relative $\left(\mathrm{ml} \cdot \mathrm{kg}^{-1} \cdot \mathrm{min}^{-1}\right)$ and absolute $(1 / \mathrm{min}) \mathrm{VO}_{2 \max }$, respectively $(p<0.01)$. The ratio of forced expiratory volume and forced vital capacity $\left(\mathrm{FEV}_{1}: \mathrm{FVC}\right)$ was $80.5 \pm 7.6,78.3 \pm 6.9$, $77.8 \pm 7.8$ and $80.1 \pm 8.4 \%$ in the NFG, IFG, ADA and NDDG groups for all subjects, respectively $(p=$ N.S.). There was no statistically significant relation between $\mathrm{FEV}_{1}$ : $\mathrm{FVC}$ and $\mathrm{VO}_{2 \max }$.

Blood lipids. Blood lipid data show that fasting total cholesterol was $5.27 \pm 39.0,5.64 \pm 1.07,5.64 \pm 1.07$ and $5.74 \pm 1.53 \mathrm{mmol} / \mathrm{l}$ in the NFG, IFG, ADA and NDDG groups, respectively (Table 2). Fasting total cholesterol and LDL cholesterol in NFG were lower $(p<0.05)$ than in all other groups. Triglycerides and total: HDL cholesterol ratios were higher in NDDG than in all other groups $(p<0.05)$ and HDL cholesterol was lower in NDDG than in all other groups $(p<0.05)$.

Blood pressure. Resting systolic and diastolic blood pressure were higher in IFG, ADA and NDDG groups in than the NFG group (Table $3, p<0.05$ ). Furthermore, resting systolic blood pressures were higher in the IFG than in the NFG group $(p<0.05)$. There were no statistically significant differences in blood pressure between the ADA and NDDG groups. Peak systolic and diastolic blood pressures obtained during exercise were higher in the IFG, ADA and NDDG groups than the NFG groups $(p<0.05)$. There were no statistically significant differences in peak blood pressure between IFG, ADA and NDDG groups (Table 3).

\section{Discussion}

Medical expenditures for Type II diabetes and associated cardiovascular diseases have increased substantially between 1970 and 1997 . Type II diabetic patients have a higher incidence of hypertension, obesity, low aerobic capacity and adverse blood lipid profiles than non-diabetic subjects [14-16]. This metabolic profile of poor insulin sensitivity, hypertension and obesity substantially increases the risk of cardiovascular disease, peripheral neuropathy, and renal failure $[1,15,17-19]$. It is therefore very important for Type II diabetes to be detected as early as possible. In 1997 the American Diabetes Association (ADA) developed new diagnostic criteria for diabetes based on fasting plasma glucose and reclassified diabetes to a more stringent fasting plasma glucose criteria of $7.0 \mathrm{mmol} / \mathrm{l}$ or more, instead of the previously used $7.8 \mathrm{mmol} / \mathrm{l}$ or more. This is more in line with the 1979 NDDG and 1985 World Health Organization (WHO) 2-h OGTT plasma glucose cut-off of $11.1 \mathrm{mmol} / \mathrm{l}$ when taking into account the degree of hyperglycaemia and the risks of microvascular and macrovascular disease [1].

The change from the NDDG to ADA classification systems resulted in a large number of subjects newly diagnosed with diabetes based on fasting plasma glucose. When we compared the ADA to the NDDG guidelines based on fasting plasma glucose, 
there was a nearly $50 \%$ increase in the number of diabetic subjects in our study group of about 3700 men. Based on previous research we anticipated that the newly classified diabetic subjects would have a similar risk factor status to those who were previously classified as diabetic (fasting glucose $\geq 7.8 \mathrm{mmol} / \mathrm{l}$ ) by the NDDG. Few studies, however, have examined this reclassification system to determine if this is indeed the case. It had been suggested that the ADA criteria would lead to earlier diagnosis of diabetes in subjects suseptible to the disease because of the finding of a study of 8096 residents of Olmsted County, Minnesota, who were 40 years old, or older, and who progressed from a non-diabetic to ADA diabetes 7 years earlier than the progression to diabetes defined by the NDDG [2]. This observation that the ADA criteria could be used for earlier diagnosis was consistent with other data obtained by comparing the ADA and the WHO diagnostic criteria (2-h glucose $\geq 11.1 \mathrm{mmol} / \mathrm{l}$ during a 75 -g OGTT) in a larger group [9]. A study of 2484 men and women aged 50 to 74 years reported that the prevalence of diabetes was similar under the ADA and the WHO criteria based on an OGTT; in this study, however, there was weak agreement between the glucose intolerance categories [3]. The DECODE Study Group also reported a weak agreement between the ADA and WHO classification systems. Furthermore, the ADA classification has resulted in a greater prevalence of diabetes in some, though not all, European populations. It was also reported, however, that there was an increase in the frequency of diabetes diagnosed when using the WHO criteria compared with the ADA criteria and that there was a weak agreement between glucose intolerant groups in a high-risk Mexican cohort [6]. Although some of these studies have found a discordance in the ADA classification system, based on fasting plasma glucose, and the NDDG or WHO classification systems, based on 2-h OGGT data, our data shows that the newly diagnosed diabetic subjects (ADA) are remarkably similar in body composition and circumference measures, systolic and diastolic blood pressures and maximum aerobic capacity compared with those (NDDG diabetic) who would have previously been diagnosed with diabetes based on fasting plasma glucose. Fasting plasma glucose is the focus of investigation because it provides a simple test and is the first indication for the need for further testing (OGTT) in the clinical setting.

Ample evidence supports that decreased aerobic capacity and a sedentary lifestyle are significant independent risk factors for cardiovascular disease [7, 20-22]. Furthermore, a reduced maximum aerobic capacity and a sedentary lifestyle have been associated with obesity, hypertension, dislipidaemia and hyperglycaemia [21]. Endurance training and increased physical fitness have been shown to reduce systolic and diastolic blood pressure in hypertensive subjects [23], total cholesterol and LDL cholesterol [24, 25], fat mass [26], and fasting blood glucose and insulin concentrations [20, 22, 27]. Other investigators [7] provide evidence of a substantially increased risk for all-cause mortality when the maximum aerobic capacity is below $10 \mathrm{METs}$, or $35 \mathrm{ml} \cdot \mathrm{kg}^{-1} \cdot \mathrm{min}^{-1}$ in men. In our study, the mean aerobic capacity of the IFG group $\left(36 \mathrm{ml} \cdot \mathrm{kg}^{-1} \cdot \mathrm{min}^{-1}\right)$ was just above this "threshold" of maximum aerobic capacity [7]. We found the mean maximum aerobic capacity of the ADA and NDDG groups to be nearly identical. These aerobic capacities were below the cut-off point for maximum aerobic capacity suggested previously and were lower than in the IFG and NFG groups. Although these authors [7] were tentative in assigning this cut-off, we suggest that the cutoff and the assignment of the ADA and NDDG groups into a similar category (diabetic) were justly chosen. It is not certain if those subjects who maintain their physical fitness (aerobic capacity) above this critical threshold are able to avoid developing Type II diabetes. Recent data suggests an increased risk of impaired glucose tolerance and diabetes with reduced cardiovascular fitness [22]. It was concluded that low cardiovascular fitness has the same effect on cardiovascular disease and all-cause mortality as on diabetes and other risk factors [22].

There are several factors that could affect aerobic capacity and could confound the relation between aerobic capacity and fasting plasma glucose. The most pertinent of these factors are smoking, a sedentary lifestyle, pulmonary disease and orthopedic limitations. The analyses on aerobic capacity excluded subjects with pulmonary limitations as measured during pulmonary function testing. There was a positive correlation between $\mathrm{FVC}$ and $\mathrm{FEV}_{1}$ with $\mathrm{VO}_{2 \max }$, although there was no correlation between $\mathrm{FEV}_{1}$ : $\mathrm{FVC}$ ratio and $\mathrm{VO}_{2 \max }$. None of the subjects listed orthopedic limitation as the reason for terminating the $\mathrm{VO}_{2 \max }$ test. Conclusions also did not differ when data on aerobic capacity were analysed only on nonsmokers, compared with data analysed for all subjects (smokers and non-smokers). It is therefore unlikely that smoking status, orthopedic limitations or pulmonary disease influenced the results according to maximum aerobic capacity. The maximum aerobic capacity is likely to reflect a sedentary lifestyle but no data on daily physical activity were collected in this group. The similarly low $\mathrm{VO}_{2 \max }$ in the ADA and NDDG groups could depend not only on reduced physical fitness and physical activity but also on reduced oxygen delivery. The pulmonary characteristics (FVC and $\mathrm{FEV}_{1}$ ) and the maximum heart rate response in both ADA and NDDG groups were lower than those of the NFG and IFG groups but were similar to one another, providing further evidence that the ADA and NDDG groups were similar. 
The degree of adiposity (as measured by body composition analysis or BMI) is associated with cardiovascular disease and cancer [28]. The degree of obesity is also directly related to fasting plasma glucose over a wide range of body composition in non-diabetic subjects [29]. Furthermore, considerable reductions in body fat result in greatly reduced fasting plasma glucose, with large amounts of weight loss, following gastric bypass surgery, resulting in the normalization of fasting plasma glucose in diabetic subjects [30]. The similarity of the ADA and NDDG groups in body composition and anthropometric data supports classifying subjects with fasting plasma glucose of $7.0 \mathrm{mmol} / \mathrm{l}$ or more (ADA group) as diabetic.

Mean-resting and peak-exercise systolic and diastolic blood pressures were also very similar in the ADA and NDDG groups $(136 \mathrm{mmHg}$ systolic and $85 \mathrm{mmHg}$ diastolic resting blood pressure, $200 \mathrm{mmHg}$ systolic and $86 \mathrm{mmHg}$ diastolic peak exercise blood pressure for both groups). This range of blood pressures at rest is considered to be borderline hypertension and all of these pressures were significantly higher than those of the NFG and IFG groups $(p<0.05)$. Hypertension has been shown to be an independent risk factor for cardiovascular disease, stroke and myocardial infarction $[31,32]$. The similarity between the ADA and NDDG groups and the statistically significant difference in the blood pressures in these groups compared with the IFG and NFG groups provides further justification for joining these groups into one diabetic group, as proposed by the ADA guidelines [1].

Our ADA and NDDG groups were also similar in total cholesterol and LDL cholesterol concentrations; however, the NDDG group had significantly lower HDL cholesterol and a significantly higher total: HDL cholesterol ratio and plasma triglycerides than the ADA group $(p<0.05)$. These high triglyceride and cholesterol ratio values of the NDDG group compared with the ADA group most likely result from skewing of these data by subjects with very progressed diabetes. The ratio of both groups, however, the total cholesterol and the total:HDL, cholesterol are above the recommended upper limits of less than $5.2 \mathrm{mmol} / 1$ (total cholesterol) and 4.5 (total:HDL cholesterol ratio). Our blood lipid data are often similar across the NFG, IFG and ADA groups, indicating that these variables do not allow differentiation between these groups. Because the mean total cholesterol and total:HDL cholesterol ratios were out of the recommended ranges, there were many subjects in each of the groups that were at increased risk for cardiovascular disease. Other studies suggested [12] that severe peripheral arteriosclerosis exists across a wide range of fasting plasma glucose and that two of the most important risk factors for this disease include hypertension and smoking. Although blood lipids are one of the major risk factors for diabetic sub- jects, lipid profile variables and arteriosclerosis are not necessarily associated with the rise in fasting plasma glucose, across groups. These differences in blood lipid profiles indicate, however, that the ADA and NDDG groups have different cardiovascular risk profiles.

Even the impaired fasting glucose group (IFG: glucose $6.1-7.0 \mathrm{mmol} / \mathrm{l}$ ) is different from the normal group (NFG:glucose $<6.1 \mathrm{~g} / \mathrm{dl}$ ) according to nearly every variable measured. This emphasizes the importance of keeping fasting plasma glucose as low as possible. This proposal was initiated by data indicating that there was a threefold greater risk of progressing to overt diabetes in subjects with initial fasting plasma glucose between 5.6 and $6.1 \mathrm{mmol} / 1$ compared with subjects with initial fasting plasma glucose of less than $5.6 \mathrm{mmol} / \mathrm{l}$ [2]. These authors therefore suggested that the upper limit of normal fasting plasma glucose be reconsidered [2].

The profiles within our study group in terms of aerobic capacity, resting and peak exercise blood pressures and body composition have been found to be similar in subjects with fasting plasma glucose between 7.0 and $7.8 \mathrm{mmol} / \mathrm{l}$ (ADA) and subjects with fasting plasma glucose of $7.8 \mathrm{mmol} / \mathrm{l}$ or more (NDDG). These results support lowering the cut-off of plasma glucose to $7.0 \mathrm{mmol} / \mathrm{l}$ or more for classifying diabetes as instituted by the American Diabetes Association in 1997. The ADA and NDDG groups, however, might not be identical in the risk of cardiovascular disease because of differences in circulating HDL cholesterol, total:HDL cholesterol ratio and triglycerides.

\section{References}

1. The expert committee on the diagnosis and classification of Diabetes Mellitus (1997) Report of the expert committee on the diagnosis and classification of Diabetes Mellitus. Diabetes Care; 20: 1183-1197

2. Dinneen SF, Maldonado D, Leibson CL et al. (1998) Effects of changing diagnostic criteria on the risk of developing diabetes. Diabetes Care 21: 1408-1413

3. de Vegt F, Dekker JM, Stehouwer CD, Nijpels G, Bouter LM, Heine RJ (1998) The 1997 American Diabetes Association criteria versus the 1985 World Health Organization criteria for the diagnosis of abnormal glucose tolerance: poor agreement in the Hoorn Study. Diabetes Care 21: 1686-1690

4. Peters AL, Schriger DL (1998) The new diagnostic criteria for diabetes: the impact on management of diabetes and macrovascular risk factors. Am J Med 105: 15S-19S

5. Anonymous (1998) Will new diagnostic criteria for diabetes mellitus change phenotype of patients with diabetes? Reanalysis of European epidemiological data. DECODE Study Group on behalf of the European Diabetes Epidemiology Study Group. BMJ 317: 371-375

6. Gomez-Perez FJ, Aguilar-Salinas CA, Lopez-Alvarenga JC, Perez-Jauregui J, Guillen-Pineda LE, Rull JA (1998) Lack of agreement between the World Health Organiza- 
tion Category of impaired glucose tolerance and the American Diabetes Association category of impaired fasting glucose. Diabetes Care 21: 1886-1888

7. Blair SN, Kohl HW 3rd, Paffenbarger RS, Clark DG, Cooper KH, Gibbons LW (1989) Physical fitness and all-cause mortality: A prospective study of healthy men and women. JAMA 262: 2395-2401

8. Harris MI, Flegal KM, Cowie CC et al. (1998) Prevalence of diabetes, impaired fasting glucose, and impaired glucose tolerance in U.S. adults. The Third National Health and Nutrition Examination Survey, 1988-1994. Diabetes Care 21: 518-524

9. Harris MI, Eastman RC, Cowie CC, Flegal KM, Eberhardt MS (1997) Comparison of diabetes diagnostic categories in the U.S. population according to 1997 American Diabetes Association and 1980-1985 World Health Organization diagnostic criteria. Diabetes Care 20: 1859-1862

10. Brozek J, Grande F, Anderson JT, Keys A (1963) Densitometric analysis of body composition: revision of some quantitative assumptions. Ann NY Acad Sci 110: 113-140

11. Pollock ML, Bohannon RL, Cooper KH et al. (1976) A comparitive analysis of four protocols for maximum treadmill stress testing. Am Heart J 92: 39-46

12. Beach KW, Brunzell JD, Strandness DE Jr (1982) Prevalence of severe arteriosclerosis obliterans in patients with Diabetes Mellitus: relation to smoking and form of therapy. Arteriosclerosis 2: 275-280

13. Beach KW, Strandness DE Jr (1980) Arteriosclerosis obliterans and associated risk factors in insulin-dependent and non-insulin dependent diabetes. Diabetes 29: 882-888

14. Tuck ML, Stern N (1992) Diabetes and hypertension. J Cardiovasc Pharmacol 19 [Suppl]: S8-18

15. Maheux P, Jeppesen J, Sheu WH et al. (1994) Additive effects of obesity, hypertension, and type 2 diabetes on insulin resistance. Hypertension 24: 695-698

16. Bjorntorp P (1996) The origins and consequences of obesity. Diabetes. Ciba Foundation Symposium 201: 68-80; 80-89; 188-193

17. Lemne C, Efendic S, Hamsten A, De Faire U (1994) Impaired glucose and insulin metabolism in borderline hypertension. Blood Press 3: 287-294

18. Mykkanen L, Haffner SM, Ronnemaa T, Bergman RN, Laakso M (1997) Low insulin sensitivity is associated with clustering of cardiovascular disease risk factors. Am J Epidemiol 146: 315-321

19. Henricsson M, Gottsäter A, Jeppsson JO, Fernlund P, Sundkvist G (1998) The frequency and severity of retinopathy are related to $\mathrm{HbA} 1 \mathrm{c}$ values after, but not before, diagnosis of NIDDM. J Intern Med 244: 149-154
20. Eriksson KF, Lindgarde F (1996) Poor physical fitness, and impaired early insulin response but late hyperinsulinaemia, as predictors of NIDDM in middle-aged Swedish men. Diabetologia 39: 573-579

21. Fletcher GF, Balady G, Blair SN et al. (1996) Statement on exercise: benefits and recommendations for physical activity programs for all Americans. A statement for health professionals by the Committee on Exercise and Cardiac Rehabilitation of the Council on Clinical Cardiology, American Heart Association. Circulation 94: 857-862

22. Wei M, Gibbons LW, Mitchell TL, Kampert JB, Lee CD, Blair SN (1999) The association between cardiorespiratory fitness and inpaired fasting glucose and type 2 diabetes mellitus in men. Ann of Intern Med 130: 89-96

23. Hagberg JM, Montain SJ, Martin WH, Ehsani AA (1989) Effect of exercise training in 60- to 69-year-old persons with essential hypertension. Am J Cardiol 64: 348-353

24. Thomas TR, Ziogas G, Harris WS (1997) Influence of fitness status on very-low-density lipoprotein subfractions and lipoprotein(a) in men and women. Metabolism 46: 1178-1183

25. Kukkonen-Harjula K, Laukkanen R, Vuori I et al. (1998) Effects of walking training on health-related fitness in healthy middle-aged adults-a randomized controlled study. Scand J Med Sci Sports 8: 236-242

26. Israel RG, Sullivan MJ, Marks RH, Cayton RS, Chenier TC (1994) Relation between cardiorespiratory fitness and lipoprotein(a) in men and women. Med Sci Sports Exerc 26: 425-431

27. Heath GW, Gavin JR 3rd, Hinderliter JM, Hagberg JM, Bloomfield SA, Holloszy JO (1983) Effects of exercise and lack of exercise on glucose tolerance and insulin sensitivity. J Appl Physiol 55: 512-517

28. Saper AG (1996) Obesity and cardiovascular disease. Ciba Foundation Symposium 201: 90-103

29. Spiegelman D, Israel RG, Bouchard C, Willet WC (1992) Absolute fat mass, percent body fat, and body-fat distribution: which is the real determinant of blood pressure and serum glucose? Am J Clin Nutr 55: 1033-1044

30. Pories WJ, MacDonald KG, Morgan EJ et al. (1992) Surgical treatment of obesity and its effect on diabetes: 10-y follow-up. Am J Clin Nutr 55: 582S-585S

31. Himmelmann A, Hedner T, Hansson L, O'Donnell CJ, Levy D (1998) Isolated systolic hypertension: an important cardiovascular risk factor. Blood Press 7: 197-207

32. Hennekens CH (1998) Lessons from hypertension trials. Am J Med 104: 50S-53S 